\title{
BMJ Open Preventing avoidable incidents leading to a presentation to the emergency department (ED) by older adults with cognitive impairment: protocol for a scoping review
}

\author{
Véronique Provencher, ${ }^{1,2}$ Mélissa Généreux, ${ }^{2,3}$ Mireille Gagnon-Roy, ${ }^{2}$ \\ Nathalie Veillette, ${ }^{4,5}$ Mary Egan, ${ }^{6,7}$ Marie-Josée Sirois, ${ }^{8,9}$ Francis Lacasse, ${ }^{1}$ \\ Kathy Rose, ${ }^{2}$ Stéphanie Stocco ${ }^{2}$
}

To cite: Provencher V, Généreux M, Gagnon-Roy M, et al. Preventing avoidable incidents leading to a presentation to the emergency department (ED) by older adults with cognitive impairment: protocol for a scoping review. BMJ Open 2016;6:e009818.

doi:10.1136/bmjopen-2015009818

- Prepublication history for this paper is available online. To view these files please visit the journal online (http://dx.doi.org/10.1136/ bmjopen-2015-009818).

Received 26 August 2015 Revised 23 November 2015 Accepted 9 December 2015

CrossMark

For numbered affiliations see end of article.

Correspondence to Professor Véronique Provencher; veronique.provencher@ usherbrooke.ca

\section{ABSTRACT}

Introduction: Older adults with cognitive impairment represent a large portion (21-42\%) of people (65+) who consult at an emergency department (ED). Because this sub-group is at higher risk for hospitalisation and mortality following an ED visit, awareness about 'avoidable' incidents should be increased in order to prevent presentations to the ED due to such incidents. This study aims to synthetise the actual knowledge related to 'avoidable' incidents (ie, traumatic injuries, poisoning and other consequences of external causes) (WHO, 2016) leading to ED presentations in older people with cognitive impairment.

Methodology and analysis: A scoping review will be performed. Scientific and grey literature (19962016) will be searched using a combination of key words pertaining to avoidable incidents, ED presentations, older adults and cognitive impairment. A variety of databases (MEDLINE, CINAHL, Ageline, SCOPUS, ProQuest Dissertations/theses, EBM Reviews, Healthstar), online library catalogues, governmental websites and published statistics will be examined. Included sources will pertain to community-dwelling older adults presenting to the $E D$ as a result of an avoidable incident, with the main focus on those with cognitive impairment. Data (eg, type, frequency, severity, circumstances of incidents, preventive measures) will be extracted and analysed using a thematic chart and content analysis.

Discussion and dissemination: This scoping review will provide a picture of the actual knowledge on the subject and identify knowledge gaps in existing literature to be filled by future primary researches. Findings will help stakeholders to develop programmes in order to promote safe and healthy environments and behaviours aimed at reducing avoidable incidents in seniors, especially those with cognitive impairment.

\section{Strengths and limitations of this study}

- Follows a rigorous process, guided by two experienced librarians and reviewed by experienced researchers, clinicians and stakeholders.

- Includes a variety of relevant sources (scientific and grey literature), which will give a clear and adequate view of the current situation.

- The partnership and collaboration with knowledge users (Public Health Department, senior home care and services stakeholders, regional Alzheimer's Society) will facilitate the interpretation, dissemination and utilisation of generated results to prevent 'avoidable' ED visits associated with incidents in older adults with cognitive impairment.

- Some incidents may not have been included in the 'avoidable' incidents and therefore not discussed.

- The results retrieved from different studies may not be applicable to every country, because of the differences in healthcare systems and culture.

\section{INTRODUCTION}

In Canada, as in many other countries, older adults (aged 65 and over) are 1.5-times more likely to consult at an emergency department (ED) than those aged 20-64. ${ }^{1}$ Among these elders, a high proportion (21-42\%) present with cognitive impairment, whether or not linked to dementia. ${ }^{2-5}$ Avoidable incidents, which may refer to unintentional injuries due to falls, motor vehicle traffic crashes, toxic substances, fire/hot objects, or other external causes, ${ }^{6}$ represent a large portion (over $20 \%$ in the USA) of ED consultations by seniors. ${ }^{7}$ In the current context of an aging population, various preventive 
measures (eg, fall prevention programmes and driving classes for seniors) have been implemented to reduce these avoidable incidents in community-dwelling seniors. ${ }^{8-10}$ Such preventive measures may, however, not be tailored to the needs of older adults with cognitive impairment, as they do not specifically target this vulnerable population. ${ }^{8-10}$ As seniors with cognitive impairment are particularly at risk of hospitalisation and mortality following an ED visit, ${ }^{2}{ }^{11}$ appropriate preventive care and services should therefore be offered to them in order to reduce the number of ED presentations, especially those associated with avoidable incidents. ${ }^{11}$

It is hypothesised that older adults with cognitive impairment are at higher risk for avoidable injuries, due to judgment errors and behaviours of self-neglect in everyday activities (eg, burning themselves while cooking due to forgetting to turn off electrical appliances, fallrelated injury after a risky transfer, poisoning after eating spoiled food in the refrigerator). ${ }^{12}{ }^{13}$ However, little is known as to whether some of these avoidable incidents leading to ED presentations are more frequent and serious in older adults with cognitive impairment, compared to the elderly population in general. Better knowledge about the type, frequency and severity of avoidable incidents that cause older people with cognitive impairment to present to the ED compared to the population in general, and the circumstances in which these incidents occur (eg, time, location, activities performed, potential causes) will help to identify preventive measures that should thus be implemented upstream in order to reduce 'avoidable' presentations to the ED in this subgroup. The study thus aims at synthetising the actual knowledge related to avoidable incidents leading to ED presentations by older people with cognitive impairment living in the community. More specifically, our purpose is to map the extent of existing literature on type, frequency, severity and circumstances of avoidable incidents in this population, as well as potential preventive measures aimed at reducing them.

\section{METHODOLOGY AND ANALYSIS}

A scoping review will be performed according to the six stages described by Arksey and O'Malley ${ }^{14}$ and revisited by Levac et al. ${ }^{15}$ Scoping review refers to a preliminary attempt to provide an overview of evidence about a topic to: (A) examine the breadth and depth of actual knowledge on the subject, (B) determine the feasibility to conduct a systematic review, (C) synthesise and disseminate research results and/or (D) identify knowledge needs to be met by future primary researches. ${ }^{14} 15$ This methodology differs from other reviews as it may include a large variety of study designs and does not require assessment of the study quality. ${ }^{14}{ }^{15}$ The selection of this methodology thus appears appropriate to map the extent and range of existing knowledge emerging from various publication types on avoidable incidents in older adults, especially those with cognitive impairments. To our knowledge, no other scoping review has been performed on this topic.

\section{Stage 1: Identifying the research question}

Preventing avoidable incidents in older adults by promoting safe and healthy environments and behaviours has been identified as one of the main priorities by the Public Health Directory in the province of Quebec (Canada). This scoping review thus aims to answer: "What is the actual knowledge on "avoidable" incidents leading to presentation to ED by older adults living in the community, particularly those with cognitive impairment, in order to implement preventive measures that are tailored to their needs?' The following specific research questions were jointly determined by regional stakeholder (MG) and researchers (VP, NV, M-JS):

1. What are the main types of avoidable incidents associated with presentations to the ED by seniors with cognitive impairment, compared to the aged population in general (eg, fall-related injuries, food poisoning, heat stroke)?

2. Are they more or less frequent and serious compared to older adults without cognitive impairment (based on Canadian data sources)?

3. Do they occur in specific circumstances (eg, during the day/night, in the summer/winter, indoor/ outdoor, when driving/cooking)?

4. Could they have been avoided by safe and healthy environments or behaviours?

For the purpose of this review, incident is defined as (A) physical injury to self or other, property loss, or property damage; and (B) caused by orientation disorder (time, person, location), self-neglect or neglect by others. ${ }^{10}{ }^{16}$ More specifically, avoidable incidents refer to traumatic injuries (eg, hip, wrist), poisoning (eg, inadvertent medication overdose, biological substances) and certain other consequences of external causes (eg, frostbite, burn, heat stroke), based on the International Classification of Injuries. ${ }^{6}$

\section{Stage 2: Identifying the relevant sources}

The research strategy has been established and validated by two experienced librarians (FL and KR) in collaboration with members of the research team (VP, MG, ME, M-JS, NV, MGR and SS). Controlled and natural keywords are displayed in table 1 . Scientific literature (1996-2016) will be searched using a variety of databases (MEDLINE, CINAHL, Ageline, SCOPUS, ProQuest Dissertations/theses, EBM Reviews, Healthstar) and online library catalogues (Institut Universitaire de Gériatrie de Montréal (IUGM), Réseau Santécom, Library and Quebec national archives). Grey literature (theses, memoirs, acts of congress, governmental or academic publications, and official statistics published by the Canadian Institute for Health Information, the National Ambulatory Care Reporting System and the Canadian Community Health Survey) will also be 
Table 1 Concepts and keywords

\begin{tabular}{|c|c|}
\hline Concept & Controlled and natural keywords \\
\hline Avoidable Incident & 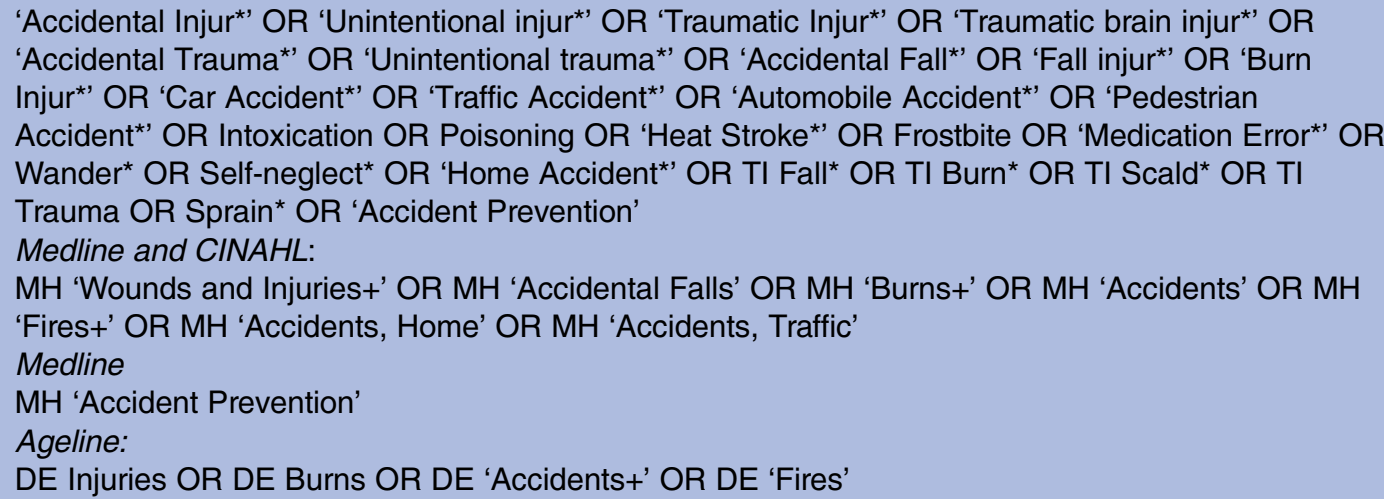 \\
\hline Older adults & $\begin{array}{l}\text { Senior* OR Elder* OR 'Older Adult`’ OR Old People OR 'Old Age' OR 'Geriatric Patient”' } \\
\text { Medline and CINAHL: } \\
\text { MH 'Aged+' }\end{array}$ \\
\hline $\begin{array}{l}\text { Emergency department } \\
\text { presentations }\end{array}$ & 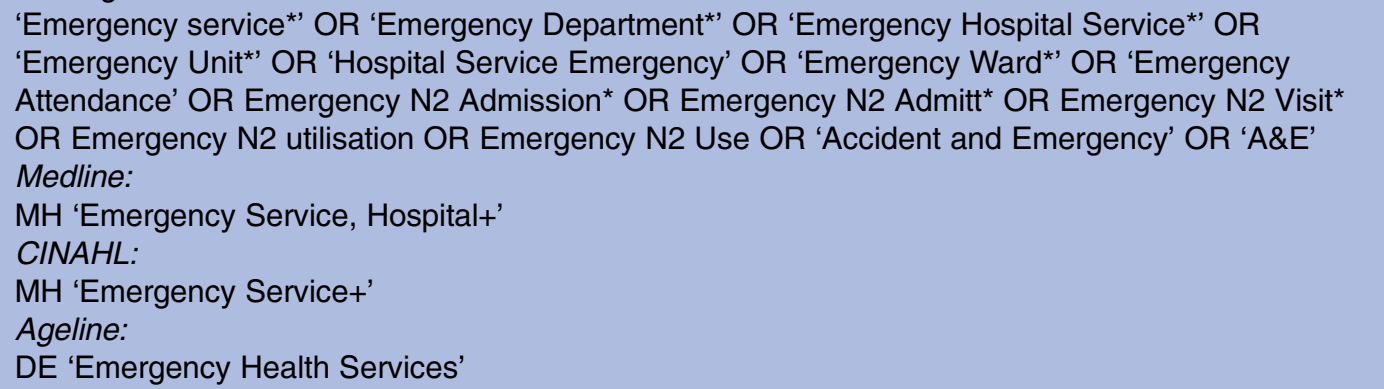 \\
\hline Cognitive impairment & $\begin{array}{l}\text { 'Cognitive decline' OR 'Cognitive* Impair*' OR 'Cognitive Deficit”' OR 'Cognitive Dysfunction*' OR } \\
\text { 'Cogniti Disorder” OR Dement* OR 'Alzheimer* disease' } \\
\text { Medline and CINAHL: } \\
\text { MH 'Dementia+' OR MH 'Cognition Disorders+' } \\
\text { Ageline: } \\
\text { DE 'Cognitive Impairment' OR DE Dementia }\end{array}$ \\
\hline
\end{tabular}

searched with the main focus on Canadian data sources. Additionally, manual research of bibliographies will be performed and key authors will be contacted for complementary information. Results from databases and grey literature will be exported to reference manager software (Zotero). Duplicates will be eliminated, and only sources in English and French will be kept.

\section{Stage 3: Selecting the studies}

The selection of sources will be carried out entirely and independently by two research assistants (MG-R and SS). First, each research assistant will screen the articles by title, according to inclusion criteria. Sources should be included if participants are: (1) 65 years old and over; (2) living in the community (domicile, private residence, senior housing or other structured environments offering, through community-based services, more or less support to older adults to perform their daily life activities); and (3) presenting to the ED as a result of an avoidable incident. Even if the focus is primarily on older adults with cognitive impairment (reported by the elders or tests, whether or not linked to dementia), relevant articles concerning those without cognitive impairment will be considered in order to point out differences in type, frequency, severity and circumstances of incidents leading to ED presentations and to identify preventive measures. Then, articles will be screened by abstract when available, and finally by full-text content. Included articles throughout the process will be compared and discussed by the two research assistants. If an agreement cannot be reached, articles will be reviewed as well by one of the researchers (VP) to reach a consensus.

\section{Stage 4: Charting the data}

When selected, articles will be carefully analysed in order to chart the data concerning the: (1) type, frequency and severity of incidents, (2) circumstances of incidents (time of the day/year, location, activities the person was engaged in at the time the incident occurred, potential causes for the incident, if available) and (3) preventive measures which may be implemented, based on evidence about formal programmes or interventions designed to prevent avoidable injuries and relevant for the older adults with cognitive impairment. In this study, data pertaining to preventive measures are included in an exploratory perspective since the study aims to recognise potential preventive measures to be 
implemented with this specific population. Data pertaining to the selected sources (year of publication, sample, country, types/research design) and the study population (type of housing, living arrangement, cognitive status, etc) will be charted (see table 2). The data charting form has been developed by the research team and will be used on Microsoft Excel. The research assistants (MGR and SS) will independently chart the data of the first 5 to 10 included articles, according to the Levac et $a l^{15}$ method. The two research assistants will then meet in order to reach a consensus that is consistent with the research question and purpose of this project. If an agreement cannot be reached, the articles will also be reviewed by one of the researchers (VP). This process will ensure high inter-rater reliability throughout data charting by one of the research assistants (MGR). Considering the iterative nature of the process, the data charting form will evolve as information is collected. New subthemes may also emerge accordingly.

\section{Stage 5: Collecting, summarising and reporting results}

Step 1: Collating and summarising

Data from included sources will be analysed using descriptive statistics and content analysis. On one hand, characteristics of included sources and study population will be collected (see table 2 for details). Main types of incidents and their frequency will be analysed using descriptive statistics (means and SD's or frequencies and percentages according to the number and type of variables). Circumstances of the incidents (eg, time, location) will be reported using descriptive results as well. On the other hand, data that pertain to severity and reasons for incidents, as well as preventive measures will be exhaustively analysed and synthetised using content analysis. ${ }^{17}$ The content analysis will be done by the research assistants (MGR and SS), and one-third co-coded by a member of the research team (VP). All narrative data will be grouped and categorised according to predetermined themes (mentioned above) and to those that will emerge during the process. Themes will be revised by team members in order to select the relevant final ones.

\section{Step 2: Reporting results}

Numerical results will be reported through graphs and tables. Narrative results will be synthetised into relevant themes. Convergences, divergences and lack in the actual knowledge will also be highlighted.

\section{Stage 6: Consulting}

Based on the partnership between researchers and knowledge users (eg, clinicians, stakeholders, community organisations), this study aims to generate relevant and applicable results in order to improve services and preventive measures for older adults, particularly those with cognitive impairment. In this context, consultation with knowledge users is essential. Specifically, the main knowledge user (MG) will be consulted throughout the process by the research director (VP) to improve the applicability of results in the actual healthcare context. Adjustments will be made according to her recommendations concerning: (1) the research question and objectives in the first stages; and (2) methodological choices (experts and pertinent reports to consult) and result

\begin{tabular}{|c|c|c|}
\hline \multicolumn{2}{|l|}{ Concepts } & \multirow{2}{*}{$\begin{array}{l}\text { Type of analysis } \\
\text { Descriptive analysis } \\
\text { (Frequency, percentage, etc) }\end{array}$} \\
\hline Sources (characteristics) & $\begin{array}{l}\text { Year of publication } \\
\text { Sample (n, age, dx) } \\
\text { Country } \\
\text { Type of sources/study design (eg, empirical, author } \\
\text { opinion) }\end{array}$ & \\
\hline Study population (Who?) & $\begin{array}{l}\text { Type of housing (domicile, private residence, seniors } \\
\text { housing or other) } \\
\text { Living arrangement (eg, living alone, with spouse or other) } \\
\text { Cognitive status (mild cognitive impairment, dementia, } \\
\text { impaired (unspecified), unimpaired, not mentioned) }\end{array}$ & $\begin{array}{l}\text { Descriptive analysis } \\
\text { (Frequency, percentage, etc.) } \\
\text { Descriptive analysis } \\
\text { (Frequency, percentage, etc) } \\
\text { Descriptive analysis } \\
\text { (Frequency, percentage, etc) }\end{array}$ \\
\hline Incidents (What?) & $\begin{array}{l}\text { Type (eg, injuries, poisoning, and other externals sources) } \\
\text { Frequency (incidence, prevalence) } \\
\text { Severity (consequences/adverse outcomes) }\end{array}$ & $\begin{array}{l}\text { Descriptive analysis } \\
\text { (Frequency, percentage, etc) } \\
\text { Content analysis (narrative) }\end{array}$ \\
\hline Circumstances (When? & Time (day/night; winter/summer) & Descriptive analysis \\
\hline Where? How and Why?) & $\begin{array}{l}\text { Location (eg, inside/outside, urban/rural areas, home/public } \\
\text { places) } \\
\text { Reasons (eg, activity the person was engaged in at the } \\
\text { time of the incident, potential causes for the incident) }\end{array}$ & $\begin{array}{l}\text { (Frequency, percentage, etc) } \\
\text { Content analysis (narrative) }\end{array}$ \\
\hline $\begin{array}{l}\text { Preventive measures } \\
\text { Formal programs or interv } \\
\text { well as relevant for older }\end{array}$ & $\begin{array}{l}\text { designed with the objective to prevent avoidable injuries as } \\
\text { vith cognitive impairment and living in the community }\end{array}$ & Content analysis (narrative) \\
\hline
\end{tabular}


interpretation (assessment of the applicability of results in the actual context of incident prevention) in intermediate stages. Meetings with research team members will be scheduled to ensure the relevance of the results and determine the most effective knowledge transfer strategies. The timeline is presented in table 3 .

\section{DISSEMINATION AND DISCUSSION}

First, planned dissemination strategies will be described. Feasibility issues, limitations and strengths will then be discussed.

\section{Dissemination}

Various dissemination strategies targeting a wide public (clinicians, stakeholders, researchers, service users, community organisations) will be used to offer services that are tailored to the needs of the elderly and to reduce the incidents leading to ED visits. Transfer knowledge strategies proposed are described in table 4, as well as possible outcomes.

\section{Feasibility}

The feasibility of this study is supported by the vast and complementary expertise of the members of the research team. Research team members have been selected in order to cover the actual knowledge concerning the population (M-JS, NV, VP, MG), the research (VP, NV, ME), the ED (NV, M-JS) as well as the methodological aspects (ME, VP, FL, KR, MG-R) and the dissemination of study conclusions (NV, VP, ME, M-JS, MG). Team members also come from a variety of fields (eg, research centre, Public Health Department, universities, etc) and have the necessary contacts and resources to facilitate knowledge transfer. The partnership and collaboration with knowledge users (Public Health Department, senior home care and services stakeholders, regional Alzheimer's Society) will facilitate the interpretation, dissemination and utilisation of generated results to prevent 'avoidable' ED presentations associated with incidents in older adults with cognitive impairment. Furthermore, the present project has clear objectives and six distinct stages which will help to complete the study within the timeline and through the financial and human resources provided by our University and Research Centre (access to a large number of databases and grey literature, help provided by two experienced librarians, with the research assistants' work supported by institutional funding).

\section{Limitations}

Some difficulties may impede the realisation as well as the completion of this study. Many strategies will thus be used to reduce their impact. First, included data may be too few to address each research question. However, this possible result is not incompatible with the objectives of conducting a scoping review, because this methodology

\section{Table 3 Schedule and contribution of the team members}

\begin{tabular}{|c|c|c|c|c|c|c|c|c|c|c|c|}
\hline \multirow[b]{2}{*}{ Stage } & \multirow[b]{2}{*}{ Date } & \multirow[b]{2}{*}{ Planned actions } & \multicolumn{9}{|c|}{ Contribution } \\
\hline & & & $\overline{\text { VP }}$ & MG & ME & FL & KR & M-JS & NV & MG-R & SS \\
\hline \multirow[t]{2}{*}{1} & $\begin{array}{l}\text { January } 2016- \\
\text { February } 2016\end{array}$ & $\begin{array}{l}\text { Development of the research question and } \\
\text { objectives }\end{array}$ & $x$ & $x$ & $x$ & & & $x$ & $x$ & & \\
\hline & & $\begin{array}{l}\text { Preliminary research and identification of } \\
\text { keywords }\end{array}$ & $x$ & & & $x$ & $\mathrm{x}$ & & & $\mathrm{x}$ & $x$ \\
\hline \multirow[t]{2}{*}{2} & & $\begin{array}{l}\text { Consultation with experts and stakeholders } \\
\text { (DPH) to have access to statistics and } \\
\text { relevant data }\end{array}$ & $x$ & $x$ & $x$ & & & $x$ & $x$ & & \\
\hline & & Team meeting ( January) & $x$ & $x$ & $x$ & & & $x$ & $x$ & $x$ & $x$ \\
\hline 3 & $\begin{array}{l}\text { March 2016- } \\
\text { April } 2016\end{array}$ & Selection of relevant articles & $x$ & & & & & & & $x$ & $x$ \\
\hline \multirow[t]{2}{*}{$4-5$} & $\begin{array}{l}\text { May 2016- } \\
\text { August } 2016\end{array}$ & $\begin{array}{l}\text { Charting of data } \\
\text { Exposure of work progress and obstacles, } \\
\text { solicitation of team member views on } \\
\text { adjustments to be made } \\
\text { Consultation with knowledge users to } \\
\text { contextualise the results from foreign data, } \\
\text { and evaluate the relevance of integrating } \\
\text { or adapting them to the Canadian health } \\
\text { context }\end{array}$ & $x$ & $x$ & $x$ & & & $x$ & $x$ & $\mathrm{x}$ & $x$ \\
\hline & & Team meeting (June-July) & $x$ & $x$ & $x$ & & & $x$ & $x$ & $\mathrm{x}$ & $x$ \\
\hline \multirow[t]{4}{*}{6} & Sept. 2016- & Writing the scientific article & $x$ & $x$ & $x$ & & & $x$ & $x$ & & \\
\hline & October 2016 & Selection of dissemination strategies & $x$ & $x$ & $x$ & & & $x$ & $x$ & & \\
\hline & $\begin{array}{l}\text { Nov. } 2016- \\
\text { Dec. } 2016\end{array}$ & $\begin{array}{l}\text { Presentation and dissemination of results } \\
\text { (websites, conferences, webinars, articles...) }\end{array}$ & $x$ & $x$ & $x$ & & & $x$ & $x$ & $x$ & $x$ \\
\hline & & Team meeting (December) & $x$ & $x$ & $x$ & & & $x$ & $x$ & $x$ & $x$ \\
\hline
\end{tabular}


Table 4 Strategies of knowledge transfer and expected outcomes

\begin{tabular}{|c|c|c|}
\hline Target audience & Dissemination strategies & Expected outcomes \\
\hline Researchers & $\begin{array}{l}\text { Publication in an scientific } \\
\text { journal } \\
\text { Savoirs UdeS (Institutional } \\
\text { Repository) } \\
\text { Website: www. OTinED.com }\end{array}$ & $\begin{array}{l}\text { Presentation of the current knowledge on the subject } \\
\text { Identification of knowledge needs to be met by the } \\
\text { primary researches } \\
\text { Determination of the feasibility of a systematic review } \\
\text { on the incidence of serious injury by causes (with } \\
\text { sub-analysis on the basis of cognitive impairment) }\end{array}$ \\
\hline Director of Public Health & $\begin{array}{l}\text { Collaboration with the Public } \\
\text { Health Department stakeholder } \\
\text { (MG) } \\
\text { Report and recommendations }\end{array}$ & $\begin{array}{l}\text { Co-construction of a prevention programme aiming at } \\
\text { promoting safe and healthy environments and } \\
\text { behaviours to reduce avoidable incidents in seniors, } \\
\text { especially those with cognitive impairment, (road } \\
\text { security, home safety, nutrition, fall prevention, abuse } \\
\text { prevention) in collaboration with community } \\
\text { organisations, municipal services (firefighting, police } \\
\text { services) and governmental organisations (SAAQ) }\end{array}$ \\
\hline Clinicians & $\begin{array}{l}\text { Information on collaborator's } \\
\text { websites } \\
\text { Workshops and webinars } \\
\text { Publication in a professional } \\
\text { journal }\end{array}$ & $\begin{array}{l}\text { Optimal healthcare services for older adults living in } \\
\text { the community (targeting important risk assessment } \\
\text { and management, preventive interventions) }\end{array}$ \\
\hline $\begin{array}{l}\text { Community Organisations } \\
\text { (ex: Alzheimer's society) }\end{array}$ & $\begin{array}{l}\text { Collaboration with the } \\
\text { Alzheimer's Society } \\
\text { Workshop for older adults and } \\
\text { their family }\end{array}$ & $\begin{array}{l}\text { Better understanding of the behaviour at risk in elders, } \\
\text { particularly those with cognitive impairment }\end{array}$ \\
\hline
\end{tabular}

may help to examine the breadth and depth of actual knowledge on the subject and to identify the knowledge needs to be met by future primary researches. Second, some information about inclusion criteria may be lacking, such as whether older adults lived in the community or are cognitively impaired. These sources of data may be retained if they are perceived as relevant by all evaluators, but the missing information will be taken into account in the analysis performed. Third, some possibly dementia-related incidents (such as hypoglycaemia/hyperglycaemia) may have been excluded from the scoping review as they are not included in the definition of 'avoidable' incidents, based on the classification retained, even if they may be problematic with a cognitively impaired population. Fourth, the definition of main concepts may vary (eg, cognitive impairment). These variations could affect the comparison between the different sources, as one concept may not have the exact same meaning for each source. In order to facilitate data analysis, the way each study defines or measures 'cognitive impairment' will be well detailed when collating data. Furthermore, the results retrieved from different studies may not be applicable to every country, because of the differences in healthcare systems and culture. Consultation with knowledge users may help to set the context and evaluate their relevance in our own (whether to integrate or adapt them to the Canadian medical context). Finally, some relevant sources may not be accessible, or not be published. Partnership with knowledge users and stakeholder's experts will help to find relevant information from a maximum of regional, provincial and national data sources (eg, Institute of Statistics of Quebec, Canadian Institute for Health Information).

\section{Strengths}

This scoping review will provide an accurate and rigorous perspective of current knowledge concerning 'avoidable' incidents with older adults, particularly those with cognitive impairment, as well as upstream measures that may be implemented in order to prevent these incidents. It offers the opportunity to explore a variety of sources (scientific and grey literature), which may be beneficial, considering the lack of reviews on the subject. Understanding of current knowledge on the subject may consequently help identify the need for further research (eg, on medical databases, charting reviews), or the feasibility of conducting a systematic review (eg, on the incidence of serious injury, by cause -with sub analysis on the basis of cognitive impairment). Furthermore, the research team's expertise and knowledge and the partnership with stakeholders will contribute to the dissemination of results in order to offer services that are tailored to the needs of older adults. Research conclusions will then support: (1) Stakeholders (public health management) to adapt their prevention programmes to the elderly in the community, and particularly to those with cognitive impairment; (2) Clinicians and managers working with older adults in the community to maximise risk assessment and management; and (3) Community organisations to support the entourage of elderly patients in the 
community through increased knowledge about at-risk behaviours. This project will mainly support the co-construction of a prevention programme aimed at reducing 'avoidable incidents' leading to ED presentations by promoting safe and healthy environments and behaviours in seniors, especially those with cognitive impairment.

\section{CONCLUSION}

This scoping review will synthetise current knowledge on avoidable incidents (type, frequency, severity and circumstances) leading to ED presentations by older adults with cognitive impairment, as well as upstream measures that may be implemented to prevent these incidents. This study will thus help to identify knowledge gaps in existing literature to be filled by future primary researches. Increased knowledge about avoidable incidents may lead to the development and implantation by stakeholders (public health management), clinicians and community organisations of injury prevention programmes or interventions tailored to the needs of older adults, particularly those with cognitive impairment, in order to reduce 'avoidable' incidents. In the current healthcare context, these changes may help to reduce medical costs while improving the health and quality of life of older adults.

\section{Author affiliations}

${ }^{1}$ Research Centre on Aging, Eastern Townships Integrated University Centre for Health \& Social Services - Sherbrooke Hospital University Centre, Sherbrooke, Québec, Canada

${ }^{2}$ Faculty of Medicine and Health Sciences, Universite de Sherbrooke, Sherbrooke, Québec, Canada

${ }^{3}$ Public Health Directory, Eastern Townships Integrated University Centre for Health \& Social Services - Sherbrooke Hospital University Centre, Sherbrooke, Québec, Canada

${ }^{4}$ Faculty of Medicine, Universite de Montreal, Montreal, Québec, Canada

${ }^{5}$ Research Centre - Institut universitaire de gériatrie de Montréal, Montreal, Québec, Canada

${ }^{6}$ Faculty of Health Sciences, University of Ottawa, Ottawa, Ontario, Canada

${ }^{7}$ Bruyere Institute, Ottawa, Ontario, Canada

${ }^{8}$ Faculty of Medicine, Université Laval, Quebec city, Québec, Canada

${ }^{9} \mathrm{CHU}$ de Québec Research Center, Quebec city, Québec, Canada

Contributors VP, NV, ME, M-JS and MG contributed to the study concept and design. VP wrote the first version of the protocol (in French), which was critically reviewed by NV, ME, MG, M-JS and MG-R. MG-R drafted a first version of the manuscript (in English) and participated in the preliminary research, with contributions by SS, FL, and KR. Please note that FL and KR were more specifically involved in the selection of relevant keywords and databases.
Funding This work is supported by the Faculty of Medicine and Health Sciences of the University of Sherbrooke (UBR-706230).

Competing interests None declared.

Provenance and peer review Not commissioned; externally peer reviewed.

Open Access This is an Open Access article distributed in accordance with the Creative Commons Attribution Non Commercial (CC BY-NC 4.0) license, which permits others to distribute, remix, adapt, build upon this work noncommercially, and license their derivative works on different terms, provided the original work is properly cited and the use is non-commercial. See: http:// creativecommons.org/licenses/by-nc/4.0/

\section{REFERENCES}

1. Institut canadien d'information sur la santé. Health Care in Canada 2011: a focus on seniors and aging. Ottawa, Ontario: CIHI, 2011.

2. Sampson EL, Blanchard MR, Jones L, et al. Dementia in the acute hospital: prospective cohort study of prevalence and mortality. Br J Psychiatry 2009;195:61-6.

3. Gray L, Peel N, Costa A, et al. Profiles of older patients in the emergency department: findings from the interRAI Multinational Emergency Department Study. Ann Emerg Med 2013;62:467-74.

4. Clevenger CK, Chu TA, Yang Z, et al. Clinical care of persons with dementia in the emergency department: a review of the literature and agenda for research. J Am Geriatr Soc 2012;60:1742-8.

5. Sirois MJ, Émond M, Ouellet MC, et al. Cumulative incidence of functional decline after minor injuries in previously independent older Canadian individuals in the emergency department. J Am Geriatr Soc 2013:61:1661-8.

6. World Health Organization. International Classification of Diseases (ICD-10). 2016. http://apps.who.int/classifications/icd10/browse/ 2016/en\#/T66-T78

7. Albert M, McCaig LF, Ashman JJ. Emergency department visits by persons aged 65 and over: United States, 2009-2010. Hyattsville, MD: National Center for Health Statistics, 2013.

8. Robitaille $\mathrm{Y}$, Laforest S, Fournier M, et al. Moving forward in fall prevention: an intervention to improve balance among older adults in real-world settings. Am J Public Health 2005;95:2049-56.

9. Nasvadi GE. Changes in self-reported driving behaviour following attendance at a mature driver education program. Transp Res Part $F$ Traffic Psychol Behav 2007;10:358-69.

10. Korner-Bitensky N, Kua A, von Zweck C, et al. Older driver retraining: an updated systematic review of evidence of effectiveness. J Safety Res 2009;40:105-11.

11. Launay C, Haubois G, Hureaux-Huynh R, et al. [Older adults and emergency department: who is at risk of hospitalisation?]. Geriatr Psychol Neuropsychiatr Vieil 2014;12:43-9.

12. Fischer BL, Gleason CE, Gangnon RE, et al. Declining cognition and falls: role of risky performance of everyday mobility activities. Phys Ther 2014;94:355-62.

13. Tierney MC, Charles J, Naglie G, et al. Risk factors for harm in cognitively impaired seniors who live alone: a prospective study. $J$ Am Geriatr Soc 2004;52:1435-41.

14. Arksey H, O'Malley L. Scoping studies: towards a methodological framework. Int J Soc Res Methodol 2005;8:19-32.

15. Levac D, Colquhoun H, O'Brien KK. Scoping studies: advancing the methodology. Implement Sci 2010;5:69.

16. Douglas A, Letts L, Eva K, et al. Measurement of harm outcomes in older adults after hospital discharge: reliability and validity. J Aging Res 2012;2012:150473.

17. L'écuyer R. Méthodologie de l'analyse développementale de contenu. Méthode GPS et concept de soi. Québec: Presses de I'Université du Québec, 1990. 